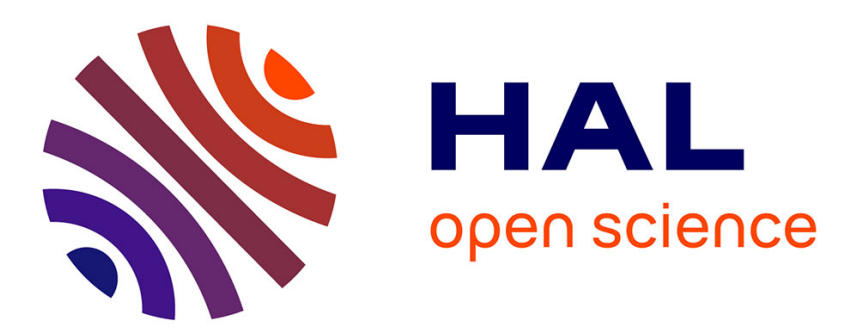

\title{
Modeling and compensation of multivariable creep in multi-DOF Piezoelectric Actuators.
}

\author{
Micky Rakotondrabe
}

\section{To cite this version:}

Micky Rakotondrabe. Modeling and compensation of multivariable creep in multi-DOF Piezoelectric Actuators.. IEEE International Conference on Robotics and Automation, ICRA'12 - Robots and Automation: Innovation for Tomorrow's Needs., May 2012, St Paul Minnesota, United States. pp.4577 - 4581. hal-00719562

\section{HAL Id: hal-00719562 https://hal.science/hal-00719562}

Submitted on 20 Jul 2012

HAL is a multi-disciplinary open access archive for the deposit and dissemination of scientific research documents, whether they are published or not. The documents may come from teaching and research institutions in France or abroad, or from public or private research centers.
L'archive ouverte pluridisciplinaire HAL, est destinée au dépôt et à la diffusion de documents scientifiques de niveau recherche, publiés ou non, émanant des établissements d'enseignement et de recherche français ou étrangers, des laboratoires publics ou privés. 


\title{
Modeling and Compensation of Multivariable Creep in multi-DOF Piezoelectric Actuators
}

\author{
Micky Rakotondrabe, Member, IEEE
}

\begin{abstract}
The scope of this paper is the modeling, identification and compensation of multivariable creep in piezoelectric actuators. Based on the inverse multiplicative scheme, we propose an approach to model and reduce the creep when the actuators have multiple degrees of freedom. The approach is simple to compute and easy to implement. The experimental results demonstrate the efficiency of the proposed approach on piezoelectric actuators.
\end{abstract}

\section{INTRODUCTION}

Piezoelectric materials are very prized for the design of actuators particularly dedicated to micro and nanopositioning. This recognition is due to the high resolution, high speed and high force density that they offer. Unfortunately, piezoelectric materials are prone to nonlinearities that make the actuators lose their accuracy. Among these nonlinearities, the creep is a phenomenon that appears at very low frequency where the accuracy is brought into play. The creep is defined as the drift appearing after the transient part when a constant input voltage is applied to the piezoelectric actuator (piezoactuator) (see Fig. 1).

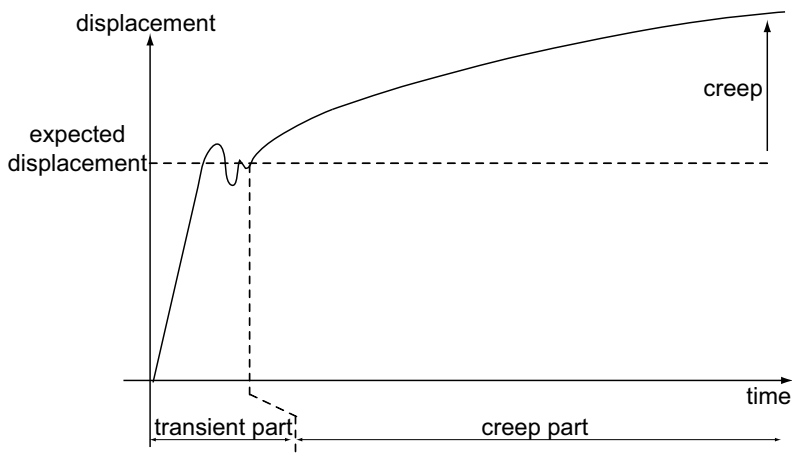

Fig. 1. Definition of the creep from a step response.

To remove the effects of the creep and to increase the accuracy of piezoactuators, closed-loop techniques (feedback) have been used [1]. These techniques also allow the consideration of the eventual model uncertainty or the parameters time dependence. However, feedback requires

FEMTO-st Institute,

UMR CNRS-6174 / UFC / ENSMM / UTBM

Automatic Control and Micro-Mechatronic Systems department (AS2M department)

25000 Besançon - France

mrakoton@femto-st.fr the use of sensors that constitutes the main limitation for micro/nanopositioning applications. Indeed, while accurate and high bandwidth sensors are very expensive and with large sizes (eg. interferometer, optical sensors), those which are embeddable on the piezoactuators are very fragile and often sensitive to noise (eg. strain gages).

To surpass the limitation of closed-loop techniques in micro/nanopositioning, open-loop techniques (feedforward) have been used. In [2], Jung et al. uses a logarithmic function to model the creep phenomenon. To compensate it, an opposite logarithmic expression is established for the applied voltage such that the output displacement will remain constant. Another approach is to model the creep as the sum of several elementary linear first order recursive equations [3][4]. The compensation is therefore based on another recursive expression computed from the initial model. In [5], the creep is modelled by a continous transfer function and its compensation is performed using the inverse model. The use of transfer functions is very interesting in an automatic point of view since the model parameters can be easily identified. However, directly inverting the model requires the bistability condition (ie. stability of the direct and of the inverse models). This is why in our previous work [6] we combine the model with a multiplicative structure to capture and compensate the creep phenomenon.

All of the above creep modeling and feedforward compensation apply for one-degree of freedom (DOF) piezoactuators, i.e. systems working on one axis. Notwithstanding, several developed piezoactuators possess multiple DOF in order to answer the requirement of several applications (x-y-z AFM scanning microscopy, 4-DOFs micromanipulation and microassembly, etc.). In these multi-DOFs piezoactuators, additionally to the creep of each axis, the coupling creep makes them very difficult to control. This paper presents the control of creep in piezoactuators that have several DOFs. Both the direct and the coupling creeps between axis are considered. Based on the modeling used in the previous work [6], we give an extension to consider multivariable creep and systems. Afterwards, we propose an identification procedure of the parameters and a multivariable compensator to delete the creep and the coupling. Finally, experiments on a real system validate the efficiency of the proposed technique.

The paper is organized as follows. In section-II, we remind the monovariable technique described in our previous work. In section-III, we present the proposed 
method to multivariable creep modeling, identification and compensation. Finally, the experimental validation is given in section-IV.

\section{REMIND OF THE MONOVARIABLE CREEP COMPENSATION}

In this section, we remind the modeling and compensation of monovariable creep as proposed in our previous work [6].

If a step voltage $U$ is applied to a (1-DOF) piezoactuator, one first obtains a displacement $y^{f}$ which corresponds to the transient part and its final value. Afterwards, a drift (the creep) denoted $y^{c}$ starts to appear (see Fig. 1). As a result, the real displacement can be modelled by $y(t)=y^{f}(t)+y^{c}(t)$ such that:

$$
y(s)=K \cdot D(s) \cdot U(s)+C(s) \cdot U(s)
$$

where $K$ is the static gain, $D(s)$ (with $D(0)=1$ ) is the dynamics for the transient part and $C(s)$ a transfer function that captures the creep evolution.

Because the term $y^{f}=K \cdot D(s) \cdot U(s)$ presents a very high dynamics (settling time less than a hundred of millisecond) relative to $y^{c}=C(s) \cdot U(s)$ (settling time: several tens of minutes), it is possible to neglect the dynamics $D(s)$ in the expression (Eq 1) and to have:

$$
y(s)=(K+C(s)) U(s)
$$

The creep compensator is defined as a transfer function such that the output $y(s)$ meets the input reference $y_{r e f}(s)$, i.e.: $y(s)=y_{r e f}(s)$. From (Eq 2), the compensator that satisfies the latter equality is:

$$
\frac{U(s)}{y^{r e f}(s)}=\frac{1}{(K+C(s))}
$$

Fig. 2 presents the block diagram of the creep model (from $(\mathrm{Eq} 1)$ ) and of its compensator (from (Eq 3)) for one DOF piezoactuators. From the figure, it is deduced that the structure of the compensator is inverse multiplicative (see for eg. [9] for more details on this structure).

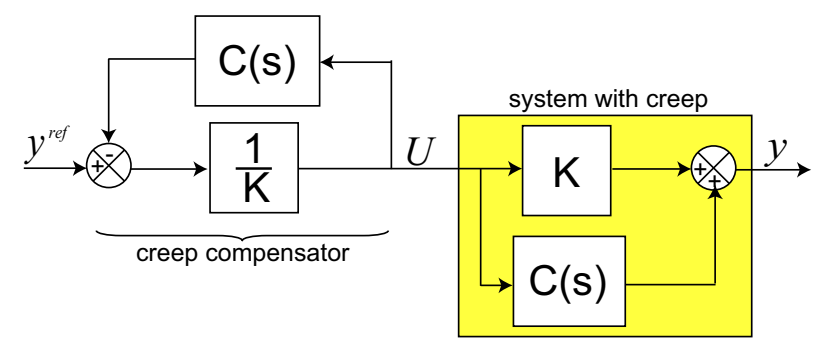

Fig. 2. Monovariable creep modeling and compensation.

\section{EXTENSION TO MULTIVARIABLE CREEP MODELING AND COMPENSATION}

\section{A. Multivariable creep modeling}

We consider now piezoactuators having multiple DOF. For that, denote $U=\left(\begin{array}{llll}U_{1} & U_{2} & \cdots & U_{n}\end{array}\right)^{T}$ and $y=$ $\left(\begin{array}{llll}y_{1} & y_{2} & \cdots & y_{n}\end{array}\right)^{T}(T$ means the transpose operator $)$ the input voltage and the output displacement respectively of the actuator. The number of DOFs of the actuator is given by $n$. Let us re-use the system's model (Eq 2) but in multivariable aspect. So we have:

- the matrix static gain defined by:

$$
K=\left(\begin{array}{llll}
K_{11} & K_{12} & \cdots & K_{1 n} \\
K_{21} & K_{22} & \cdots & K_{2 n} \\
\vdots & \vdots & \ddots & \vdots \\
K_{n 1} & K_{n 2} & \cdots & K_{n n}
\end{array}\right)
$$

where $K_{i i}$ (the diagonal) are the direct static gains relating the inputs $U_{i}$ and the outputs $y_{i}$, and $K_{i j}(i \neq j)$ are the coupling static gains relating the inputs $U_{j}$ and the outputs $y_{i}$.

- the matrix creep model defined by:

$$
C=\left(\begin{array}{llll}
C_{11} & C_{12} & \cdots & C_{1 n} \\
C_{21} & C_{22} & \cdots & C_{2 n} \\
\vdots & \vdots & \ddots & \vdots \\
C_{n 1} & C_{n 2} & \cdots & C_{n n}
\end{array}\right)
$$

where $C_{i i}$ (the diagonal) are the direct creep relating the input $U_{i}$ and the outputs $y_{i}$, and $C_{i j}(i \neq j)$ are the coupling creep relating the inputs $U_{j}$ and the outputs $y_{i}$.

Remark.1: we assume that every input $U_{i}$ has an influence to the outputs $y_{i}$, for $i=1 \cdots n$. It means that all $K_{i i}$ is non-null and therefore the inverse $K^{-1}$ exists. This assumption is naturally respected for as much as in an actuator, one must have at least a control signal dedicated to an output.

\section{B. Multivariable creep identification}

The identification procedure of parameters $K$ and $C(s)$ is as follows.

$\rightarrow$ Apply a step input $U_{1}$ to the actuator and leave the other inputs $U_{j}$ (with $j \neq 1$ ) to zero.

$\rightarrow$ Capture the curves of all outputs $y_{i}(i=1 \cdots n)$.

$\rightarrow$ If we denote $y_{i}^{f}$ the expected displacement just after transient part (see Fig. 1), each element $K_{i 1}$ is identified by using:

$$
K_{i 1}=\frac{y_{i}^{f}}{U_{1}}
$$

$\rightarrow$ To identify the creep model, one must separate the creep curve from the original response (see Fig. 1). Then, each creep model $C_{i 1}$ is identified by using a system identification technique. If $y_{i}^{c}$ is the curve of the creep, the transfer function to be identified is: 


$$
C_{i 1}(s)=\frac{y_{i}^{c}(s)}{U_{1}(s)}
$$

$\rightarrow$ To identify $K_{i j}$ and $C_{i j}(s)$ where $j \neq 1$, repeat the above sequence by applying the corresponding voltage $U_{j}$ to the actuator.

\section{Multivariable creep compensation}

From the system's model as in (Eq 2), we derive the voltage to be applied that allows the output $y$ meets the reference input $y^{\text {ref }}=\left(\begin{array}{llll}y_{1}^{r e f} & y_{2}^{\text {ref }} & \cdots & y_{n}^{\text {ref }}\end{array}\right)^{T}$ as follows:

$$
U(s)=(K+C(s))^{-1} y^{r e f}(s)
$$

After rearrangement of (Eq 2), the equation of the compensator becomes:

$$
U(s)=K^{-1}\left(y^{r e f}-C(s) U(s)\right)
$$

Remark.2: in the particular case of monovariable system, the developed compensator defined in (Eq 9) (and therefore in (Eq 8)) gives the result in (Eq 3).

Remark.3: using the proposed compensator (Eq 9), both the direct creeps and the coupling creeps and coupling gains are deleted, i.e. the compensator performs the decoupling. Furthermore, the accuracy is improved because this compensator will result $y=y^{\text {ref }}$ when replacing $U$ in (Eq 2) by (Eq 9).

Fig. 3 presents the block diagram of (Eq 9) which has an inverse multiplicative structure. The advantage is that there is no direct inversion of transfer $C(s)$ and therefore no need bi-causality condition is required for this matrix transfer function. It also means that noinvertibility condition to matrix $C(s)$ is necessary. In other words, systems without direct creep (i.e. $C_{i i}(s)=$ $0)$ can also be considered by the compensator. We remind that $K$ is invertible according to Remark.1.

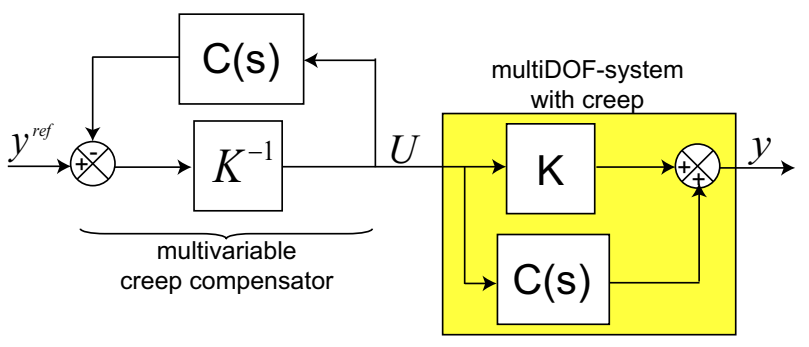

Fig. 3. Block diagram of the multivariable compensator.

\section{EXPERIMENTAL RESUlts}

In this section, we apply the proposed method to model and compensate the creep of a 2-DOFs piezoactuator.

\section{A. The experimental setup}

The 2-DOFs piezoactuator, developed in [7], is made up of two piezolayers (PZT ceramic material) with 4 local electrodes at its surfaces and one middle electrode for ground (Fig. 4-a and b). It can be assimilated to a cantilever beam clamped at one end. The two DOFs are obtained with a judicious application of voltages on the electrodes. In the figure, $P$ indicates the polarization direction of the material. The out-of-plane displacement $y_{1}$ is obtained by applying a voltage $U_{1}$ to the four electrodes (Fig. 4-c). To obtain the in-plane displacement $y_{2}$, one has to apply a voltage $U_{2}$ and its opposite $-U_{2}$ to the four electrodes (Fig. 4-d). Finally the application of both $U_{1}$ and $U_{2}$ allows to obtain a displacement in the workspace (Fig. 4-e). The piezoactuator can therefore be seen as a system with input $U=\left(\begin{array}{ll}U_{1} & U_{2}\end{array}\right)^{T}$ and output $y=\left(\begin{array}{ll}y_{1} & y_{2}\end{array}\right)^{T}$.

The experimental setup is composed of (Fig. 4-f):

- the 2-DOF piezoactuator. Its total dimension is $15 \mathrm{~mm} \times 2 \mathrm{~mm} \times 0.5 \mathrm{~mm}$

- two optical sensors to measure the two displacements $y_{1}$ and $y_{2}$ at the tip of the piezoactuator. The sensors, from Keyence (LK2420), have 10nm of resolution and $0.1 \mu \mathrm{m}$ of accuracy.

- a computer and a DSPace-board to generate control signals, to implement the compensator and to acquire measurements. The chosen sampling frequency is $50 \mathrm{~Hz}$ which is high enough to account the cutting frequency of the creep. The MatlaBSimulink ${ }^{\mathrm{TM}}$ software is used to manage the signals and for the implementation.

- a custom high-voltage (HV) amplifier having two independant lines.

\section{B. Characterization of the 2-DOF piezoactuator}

In this subsection, we characterize the piezoactuator. For that, we first apply a step voltage $U_{1}=40 \mathrm{~V}$ and set $U_{2}=0$. The resulting output $y_{1}$ and $y_{2}$ are represented in Fig. 5-a and c respectively. They clearly show the presence of the direct and coupling creeps due to $U_{1}$. Then, we apply a step input voltage $U_{2}=40 \mathrm{~V}$ and set $U_{1}=0$. As pictured in Fig. 5-b and d, the input voltage $U_{2}$ also causes a direct and a coupling creeps on $y_{2}$ and $y_{1}$ respectively. Now let us model the piezoactuator and identify the different parameters.

\section{Modeling and identification}

Using (Eq 2), the model of the 2-DOFs piezoactuator is:

$$
\begin{aligned}
& \left(\begin{array}{l}
y_{1} \\
y_{2}
\end{array}\right)=\left(\left(\begin{array}{ll}
K_{11} & K_{12} \\
K_{21} & K_{22}
\end{array}\right)\right. \\
& \left.+\left(\begin{array}{ll}
C_{11}(s) & C\left({ }_{12}(s)\right. \\
C_{21}(s) & C_{22}(s)
\end{array}\right)\right)\left(\begin{array}{c}
U_{1} \\
U_{2}
\end{array}\right)
\end{aligned}
$$




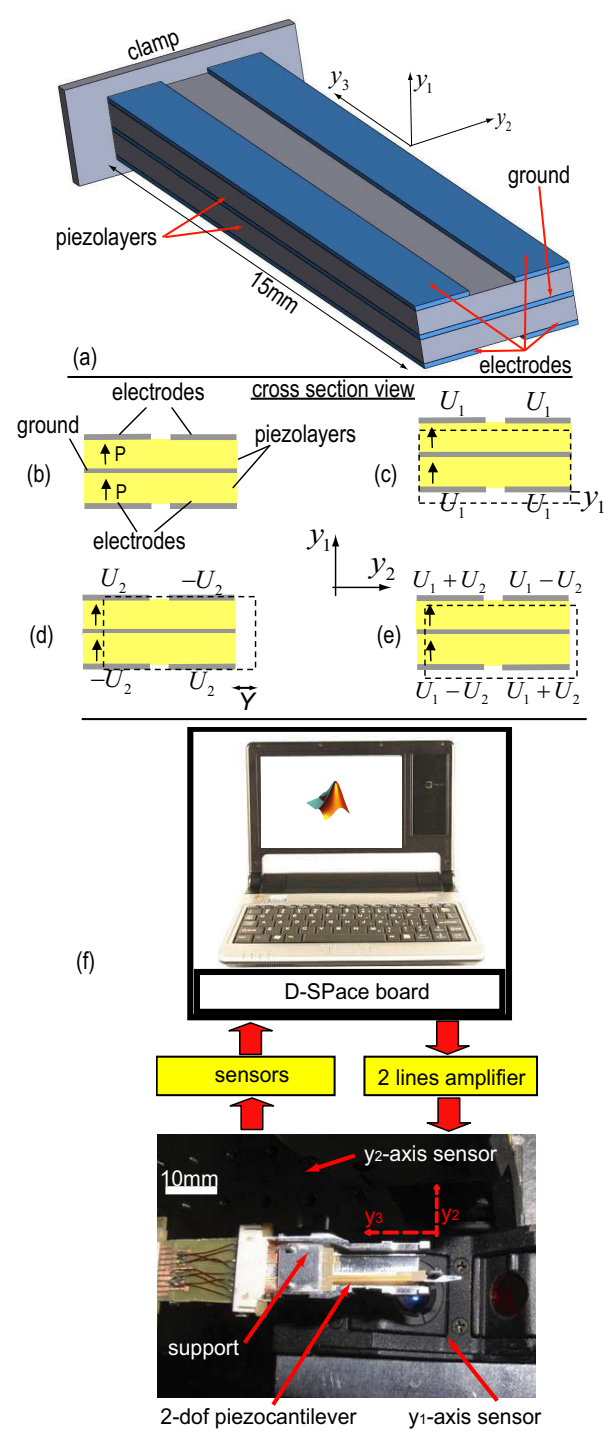

Fig. 4. (a) CAD view of the 2-DOFs piezoactuator [7]. (b) cross section view of the 2-DOFS piezoactuator. (c) achievement of $y_{1}$ displacements. (d) achievement of $y_{2}$ displacements. (e) achievement of both $y_{1}$ and $y_{2}$ displacements. (f) presentation of the experimental setup.

To identify $K$, we use the identification procedure in Section. III-B and the values after transient part issued from Fig. 5. These values are:

- the expected value $y_{1}^{f}=59 \mu m$ when applying $U_{1}=$ $40 \mathrm{~V}$ (Fig. 5-a),

- the coupling value $y_{1}^{f}=3.56 \mu \mathrm{m}$ when applying $U_{2}=$ $40 \mathrm{~V}$ (Fig. 5-b),

- the coupling value $y_{2}^{f}=4.28 \mu m$ when applying $U_{1}=$ $40 \mathrm{~V}$ (Fig. 5-c),

- and the expected value $y_{2}^{f}=27.8 \mu m$ when applying $U_{2}=40 \mathrm{~V}$ (Fig. 5-d),

We have:

$$
K=\left(\begin{array}{ll}
1.475 & 0.089 \\
0.107 & 0.695
\end{array}\right)
$$

To identify the creep $C(s)$, we separate the creep

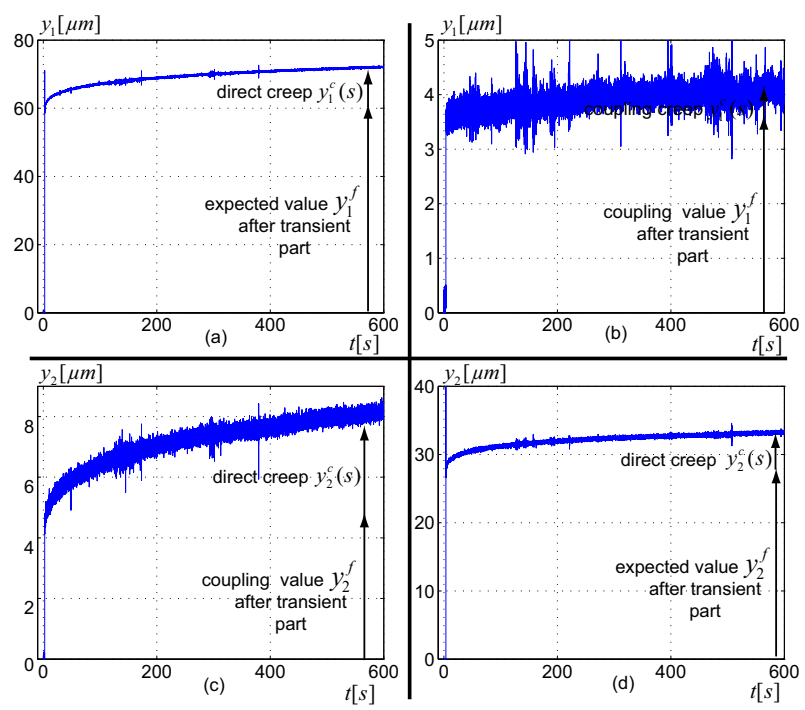

Fig. 5. Step response of the piezoactuator.

curves from Fig. 5 and we obtain the results pictured in Fig. 6.

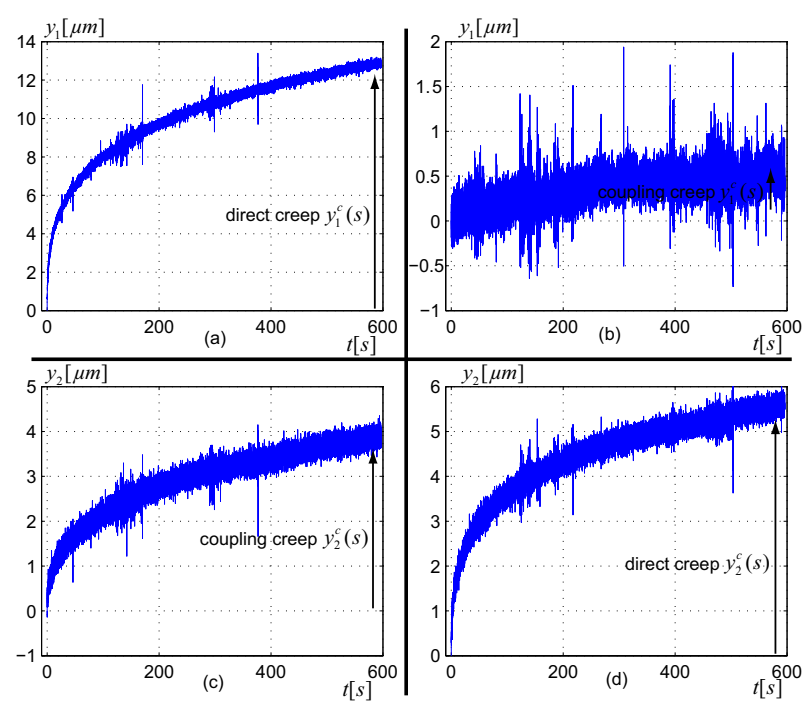

Fig. 6. Creep curves of the piezoactuator.

Then, following the creep identification procedure in Section. III-B, the different signals $y_{1}^{c}(t)$ and $y_{2}^{c}(t)$, and the ARMAX method (Autoregressive Moving Average eXogenous [8]), we obtain:

$$
\begin{aligned}
& C(s)= \\
& \left(\begin{array}{ll}
\frac{3 \times 10^{-4}(s+50)(s+0.01)}{(s+0.12)(s+0.004)} & \frac{2 \times 10^{-6}(s+0.076)\left(s^{2}+78 s+2599\right)}{(s+0.004)\left(s^{2}+4 s+7.8\right)} \\
\frac{6.2 \times 10^{-5}(s+50)(s+0.011)}{(s+0.09)(s+0.003)} & \frac{1.4 \times 10^{-4}(s+50)(s+0.01)}{(s+0.12)(s+0.004)}
\end{array}\right)
\end{aligned}
$$

Fig. 7 show the comparison of the simulation of the identified model with the experimental results. The figure show the convenience of the identified model. 

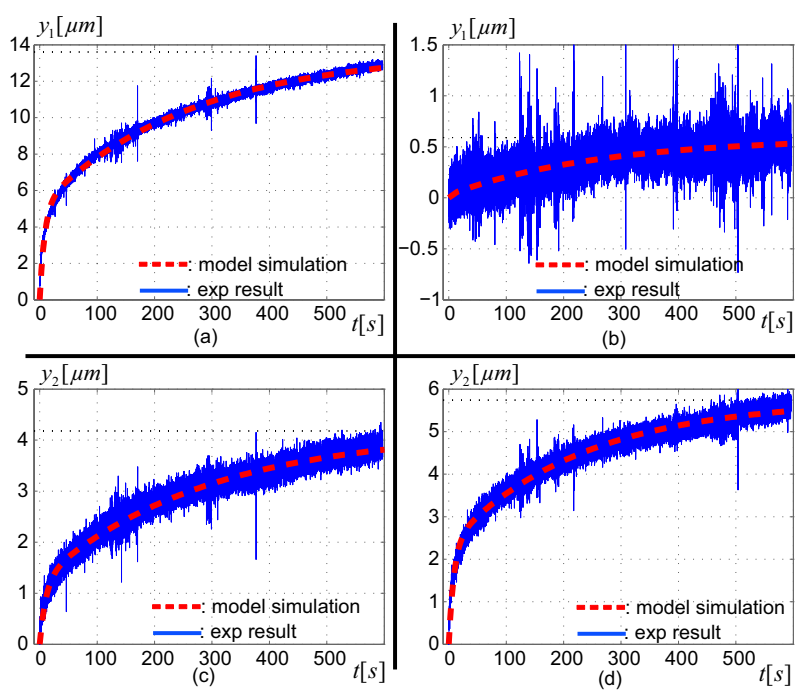

Fig. 7. Model simulation and experimental results for the creep.

\section{Compensation of the creep}

Let us now implement the creep compensator by using MATLAB-SimulinK $^{\mathrm{TM}}$ software. The implementation is based on the diagram shown in Fig. 3 and on the previously identified parameters.

First, we apply a step reference input $y_{1}^{r e f}=40 \mu \mathrm{m}$. Fig. 8-a pictures the resulting output $y_{1}$. It clearly shows that the creep has been removed and we obtain the required accuracy: $y_{1}=y_{1}^{r e f}$. If we take a look on the output $y_{2}$ (see Fig. 8-c), we remark that not only the coupling creep is removed but also the whole coupling displacement (see Remark.3). Indeed, we have $y_{2}=$ $0 \mu m$ whatever $y_{1}^{r e f}$ non-null is.

Now, we apply a step reference input $y_{2}^{\text {ref }}=20 \mu \mathrm{m}$. Once again, the creep has been removed and the accuracy was improved (Fig. 8-d): $y_{2}=y_{2}^{\text {ref }}$. On the other hand, the coupling displacement and the coupling creep have also been removed because $y_{1}=0 \mu m$ when $y_{2}^{\text {ref }}$ is nonnull.

\section{Conclusion}

This paper presented the modeling, identification and compensation of creep phenomenon in multi-DOF piezoactuators. The approach is an extension of the monovariable creep modeling and compensation presented in our previous work. An adapted identification procedure and a compensation scheme based on an inverse multiplicative scheme have therefore been proposed for the multivariable creep. The main advantages of the approach are the elimination of the creeps for every axis and the decoupling between these axis. Furthermore, the compensator is simple to compute and easy to implement since it is directly based on the identified model: as soon as the model is identified, the compensator is derived. Experimental results on a 2-DOFs piezoactuator have

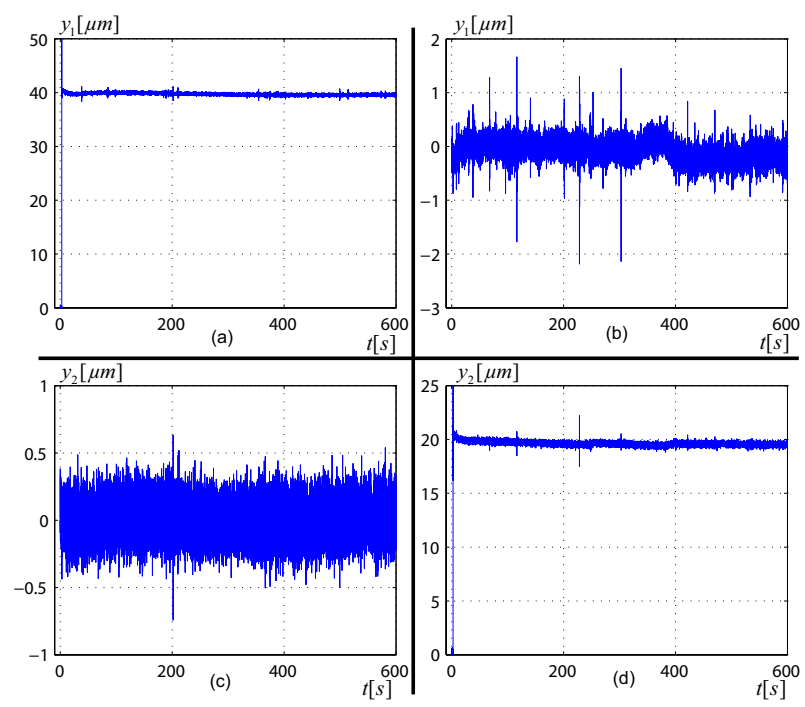

Fig. 8. Step response when using the creep compensator.

confirmed the efficiency of the proposed approach. Future works include the more in depth structural analysis of the compensator and its application to systems with higher DOFs, such as 3-DOFs piezotube actuator in atomic force microscopy.

\section{ACKNOWLEDGMENT}

This work is supported by the national project ANRMIMESYS.

\section{REFERENCES}

[1] Micky Rakotondrabe, Yassine Haddab and Philippe Lutz, "Quadrilateral modelling and robust control of a nonlinear piezoelectric cantilever", IEEE Transactions on Control Systems Technology (TCST), Vol.17(3), pp:528-539, May 2009.

[2] H. Jung, J.Y. Shim and D. Gweon, "New open-loop actuating method of piezoelectric actuators for removing hysteresis and creep", Review of Scientific Instruments, 71 (9), pp.3436-3440, 2000.

[3] K. Kuhnen and H. Janocha, "Compensation of creep and hysteresis effects of piezoelectric actuators with inverse systems", 6th Int. Conf. on New Actuators, pp:309-312, Bremen 1998.

[4] B. Mokaberi and A. A. G. Requicha, "Compensation of scanner creep and hysteresis for AFM nanomanipulation", IEEE Transactions on Automation Science and Engineering, Vol.5, $N^{o} 2$, pp.197-208, 2008.

[5] D. Croft, G. Shed and S. Devasia, "Creep, hysteresis and vibration compensation for piezoactuators: atomic force microscopy application", ASME Journal of Dynamic Systems, Measurement and Control, 2001.

[6] Micky Rakotondrabe, Cédric Clévy and Philippe Lutz, "Complete open loop control of hysteretic, creeped and oscillating piezoelectric cantilever", IEEE Transactions on Automation Science and Engineering (TASE), Vol.7(3), pp:440-450, July 2010.

[7] R. Pérez, J. Agnus, C. Clévy, A. Hubert and N. Chaillet, "Modeling, fabrication and validation of a high performances 2-DOF piezoactuator for micromanipulation", IEEE/ASME Transactions on Mechatronics (TMech), Vol.10(2), pp:161-171, April 2005.

[8] Lennart Ljung, "System identification toolbox user's guide", The Mathworks, Vol.10(2), October 2008.

[9] Bruce A. Francis, Malcolm C. Smith and Jan C. Willems, "Control of Uncertain Systems: Modelling, Approximation, and Design", A Workshop on the Occasion of Keith Glover's 60th Birthday, Springer Verlag, 2006. 